\title{
Assessing numbers and faces: a prerequisite for improving access to lymphatic filariasis morbidity care
}

\author{
Sören L. Becker ${ }^{a, b, c, *}$, Thomas Fürst ${ }^{d}$, David G. Addiss ${ }^{e}$ and Jürg Utzinger ${ }^{a, b}$ \\ ${ }^{a}$ Department of Epidemiology and Public Health, Swiss Tropical and Public Health Institute, P.O. Box, CH-4002 Basel, Switzerland; \\ ${ }^{b}$ University of Basel, P.O. Box, CH-4003 Basel, Switzerland; ' Institute of Medical Microbiology and Hygiene, Saarland University Medical \\ Center, Kirrberger Straße, Building 43, D-66421 Homburg/Saar, Germany; ${ }^{d}$ Centre for Health Policy and Department of Infectious Disease \\ Epidemiology, Imperial College London, South Kensington Campus, London, SW7 2AZ, UK; ${ }^{\circ}$ Children Without Worms, Task Force for Global \\ Health, 325 Swanton Way, Decatur, GA 30030, USA \\ *Corresponding author: Tel: +496841 16 23943; E-mail: soeren.becker@uks.eu \\ Received 27 January 2015; revised 23 February 2015; accepted 23 February 2015

\begin{abstract}
Concerted efforts to eliminate lymphatic filariasis worldwide have registered success; multiple rounds of mass drug administration have led to the interruption of transmission in many previously endemic areas. However, the management of patients with established clinical disease (e.g., lymphoedema, hydrocoele and acute dermatolymphangioadenitis) has not been addressed sufficiently. Two recent studies from Malawi underscore the need for accurate epidemiological and clinical data, and comprehensive morbidity assessments across various domains of daily life. Addressing these issues will guide the implementation of programmes to improve access to treatment and disability prevention for affected individuals in Malawi and beyond.
\end{abstract}

Keywords: Lymphatic filariasis, Morbidity management, Quality of life

Lymphatic filariasis (LF) is a mosquito-borne neglected tropical disease (NTD), caused by the nematode parasites Wuchereria bancrofti, Brugia malayi and B. timori. ${ }^{1} \mathrm{LF}$ is a high-morbidity, lowmortality NTD with an estimated global burden of 2.78 million disability-adjusted life years (DALYs). ${ }^{2,3}$ Adult filariae cause severe damage to the lymphatic system of infected people, which leads to considerable long-term morbidity. As many as 36 million individuals suffer from LF-related lymphoedema, elephantiasis and hydrocoele. Many of them regularly experience painful, erysipelaslike manifestations, commonly referred to as acute dermatolymphangioadenitis (ADLA). This condition is caused by secondary bacterial infection and accounts for much LF-related morbidity. ${ }^{4}$

In 2000, the Global Programme to Eliminate Lymphatic Filariasis (GPELF) was launched with the goal to eliminate LF as a public health problem by 2020. GPELF is built on two major pillars: 1. mass drug administration (MDA) and complementary measures (e.g., vector control) to prevent clinical disease and interrupt LF transmission; and 2. morbidity management facilitated by improved access to health care for those suffering from LF-related morbidity. ${ }^{5}$ While MDA has been implemented successfully in 60 of the 73 LF-endemic countries, data on the burden of LF-related morbidity are scarce and appropriate programmes for clinical management of $L F$ in endemic countries are relatively few. ${ }^{6}$

Two studies published in the Transactions of the Royal Society of Tropical Medicine and Hygiene in December 2014 illustrate pervasive morbidity patterns of LF and practical challenges for an accurate estimation of the LF burden in endemic settings. Research conducted by Smith and colleagues ${ }^{7}$ in the catchment area of a health centre in southern Malawi revealed that morbidity data routinely collected by community drug distributors during MDA programmes considerably underestimated the true number of lymphoedema cases. Indeed, administration of a questionnaire and subsequent clinical examination found 69 cases of lymphoedema (32 lymphoedema cases per 10000 population), while community drug distributors reported slightly less than half (33 cases). Most of the patients (94\%) had experienced ADLA at least once, and half of them reported having suffered $\geq 2$ episodes during the past 6 months. A complementary study by Martindale et al. ${ }^{8}$ employed a semi-structured questionnaire to investigate the LF-related health impact in the 69 individuals with lymphoedema. For eight distinct domains of daily life (8D), such as mobility, pain and social participation, each individual was assigned scores at five different levels ( $5 \mathrm{~L}$ ) to evaluate the perceived health impact of $\mathrm{LF}$. This $8 \mathrm{D} / 5 \mathrm{~L}$ survey revealed that most patients with lymphoedema (77\%) experienced negative economic consequences, e.g., due to reduced ability to walk or capacity to work. This loss of productivity was further pronounced during ADLA. Indeed, one-third of the patients reported missing days of work during the past 6 months due to such 'acute attacks'. Disease-related pain/ discomfort (65\%) and anxiety/depression (45\%) due to disfigured 


\section{S. L. Becker et al.}

limbs or hydrocoele were also frequently mentioned and emphasise the multifaceted impact of LF on quality of life.

Many implications for LF programmes and research arise from these two Malawian studies, and we would like to highlight two observations that are likely to be relevant in other LF-endemic countries. First, disease burden estimates depend on quality assessment of prevalence and incidence. The number of lymphoedema cases identified through a questionnaire and confirmatory clinical examination was more than twice that obtained through data routinely collected during MDA activities. The insufficient case detection rate during routine activities in the Malawian studies is particularly worrying because GPELF uses similar approaches in other LF-endemic settings for the rapid epidemiological assessment of morbidity and MDA coverage rates. It follows that there is a need to carefully evaluate the case detection strategies for LF in all endemic areas. As interruption of LF transmission has been achieved in several countries and MDA programmes are scaling down, more emphasis needs to be placed on the second pillar of GPELF, i.e., morbidity management and prevention of disability. WHO recently recommended that national programme managers 'assess the numbers of cases of ADLA, lymphoedema and hydrocoele in all implementation units' as a first step to implement morbidity management strategies. ${ }^{9}$ Knowing the numbers will enable public health programmes to identify the funds and human resources needed to reach those affected, to ensure proper training of healthcare professionals across affected communities and ultimately, to provide access to treatment and relieve patient suffering. This is of paramount importance in all LF-endemic areas as the chronic sequelae of LF will continue to cause significant morbidity in the post-transmission era. Clinical assessment is key to accurately estimating the magnitude of such 'post-transmission morbidity'.

Second, the perceived impact on various domains of patients' lives underscores the difficulty of accurately assessing the true burden that is attributable to LF and other chronic NTDs, or even the nature of that burden. It follows that aggregated burden of disease measures should be interpreted with caution. For instance, the DALY figure cited at the beginning of our commentary does not sufficiently consider manifestations of ADLA, the social and economic impact of LF due to lost working productivity, the devastating effects on mental health due to stigma and discrimination ${ }^{10,11}$ or subtle morbidity that may not easily come to clinical attention. ${ }^{12}$ To more accurately estimate the LF burden and its implications for the daily life of affected individuals, other tools such as questionnaires evaluating the health-related quality of life (HRQOL) have been proposed. ${ }^{13}$ These patient-based HRQoL assessments are often summarised in burden estimates based on quality-adjusted life years (QALYs). However, QALYS have been criticised for the high variability of self-rated health effects across different countries and cultures that minimise comparability. ${ }^{14}$

In conclusion, the studies by Smith et al. ${ }^{7}$ and Martindale et al. ${ }^{8}$ provide a detailed account of the numbers and the many faces of LF-related morbidity in southern Malawi, both of which remain all too often hidden. It is likely that the issues highlighted here are equally relevant for other LF-endemic areas, particularly the need for clinical surveys to define the extent of ADLA, lymphoedema and hydrocoele. Quality epidemiological data from different settings will shape morbidity management and disability prevention programmes, so that the 'faces behind the numbers', ${ }^{15}$ i.e., the millions of patients suffering from the aforementioned conditions, finally gain adequate access to basic health care, necessary treatment and additional support. While GPELF has succeeded in preventing an enormous number of future LF cases, the programme's overall success or failure will equally be determined by the quality of clinical care that is provided to affected individuals suffering from LF-related morbid sequelae. Future interdisciplinary research should therefore prioritise the question of how the needs of these patients can be more accurately assessed and met. In this spirit, the two studies reviewed here can be seen as a gentle reminder for any public health intervention to consider the numbers and faces of the targeted condition to most effectively help those in need.

Authors' contributions: SLB wrote the first draft of the manuscript; TF, DGA and $\mathrm{JU}$ read and critically revised the manuscript. All authors read and approved the final manuscript. SLB is the guarantor of the paper.

Funding: TF is grateful to the Swiss National Science Foundation (SNSF) for an Advanced Postdoc Mobility fellowship [project no. P300P3-154634].

Competing interests: None declared.

Ethical approval: Not required.

\section{References}

1 Utzinger J, Becker SL, Knopp S et al. Neglected tropical diseases: diagnosis, clinical management, treatment and control. Swiss Med Wkly 2012;142:w13727.

2 Murray CJL, Vos T, Lozano R et al. Disability-adjusted life years (DALYS) for 291 diseases and injuries in 21 regions, 1990-2010: a systematic analysis for the Global Burden of Disease Study 2010. Lancet 2012;380:2197-223.

3 Hotez PJ, Alvarado M, Basáñez MG et al. The Global Burden of Disease Study 2010: interpretation and implications for the neglected tropical diseases. PLoS Negl Trop Dis 2014;8:e2865.

4 Addiss DG, Brady MA. Morbidity management in the Global Programme to Eliminate Lymphatic Filariasis: a review of the scientific literature. Filaria J 2007;6:2.

5 Ichimori K, King JD, Engels D et al. Global programme to eliminate lymphatic filariasis: the processes underlying programme success. PLoS Negl Trop Dis 2014;8:e3328.

6 WHO. Global programme to eliminate lymphatic filariasis: progress report, 2013. Wkly Epidemiol Rec 2014;89:409-18.

7 Smith EL, Mkwanda SZ, Martindale S et al. Lymphatic filariasis morbidity mapping: a comprehensive examination of lymphoedema burden in Chikwawa district, Malawi. Trans R Soc Trop Med Hyg 2014;108:751-8.

8 Martindale S, Mkwanda SZ, Smith E et al. Quantifying the physical and socio-economic burden of filarial lymphoedema in Chikwawa district, Malawi. Trans R Soc Trop Med Hyg 2014;108:759-67.

9 WHO. Lymphatic filariasis: managing morbidity and preventing disability. An aide-mémoire for national programme managers. Geneva: World Health Organization; 2013. http://apps.who.int/iris/ bitstream/10665/85347/1/9789241505291_eng.pdf [accessed 23 February 2015]. 
10 Weiss MG. Stigma and the social burden of neglected tropical diseases. PLoS Negl Trop Dis 2008;2:e237.

11 Litt E, Baker MC, Molyneux D. Neglected tropical diseases and mental health: a perspective on comorbidity. Trends Parasitol 2012;28:195-201.

12 King $\mathrm{CH}$, Bertino AM. Asymmetries of poverty: why global burden of disease valuations underestimate the burden of neglected tropical diseases. PLoS Negl Trop Dis 2008;2:e209.
13 Thomas C, Narahari SR, Bose KS et al. Comparison of three quality of life instruments in lymphatic filariasis: DLQI, WHODAS 2.0, and LFSQQ. PLoS Negl Trop Dis 2014;8:e2716.

14 King CH. Health metrics for helminth infections. Acta Trop 2015;141: 150-60.

15 Addiss DG. Global elimination of lymphatic filariasis: a "mass uprising of compassion". PLoS Negl Trop Dis 2013;7:e2264. 